\title{
Stress-strain state of a three-layer rod. Comparison of the results of analytical and numerical calculations with the experiment
}

\author{
Nikita Tsybin ${ }^{1, *}$, Robert Turusov $^{1}$, Vladimir Andreev ${ }^{1}$, and Alexey Kolesnikov ${ }^{2}$ \\ ${ }^{1}$ Moscow State University of Civil Engineering, Yaroslavskoe shosse, 26, Moscow, 129337, Russia \\ ${ }^{2}$ LLC "LIRA soft"
}

\begin{abstract}
The article deals with the stress-strain state of a three-layer rod under central compression. The results of analytical calculation and numerical calculation (FEM) are compared with experimental data. It is assumed that the interaction of layers is accomplished through a contact layer. The contact layer is considered as a transversally anisotropic elastic medium with such parameters that it can be represented as a set of short elastic rods that are not connected to each other and are normally oriented to the contact surface. Such an assumption allows us to obtain an analytical solution of the problem in a closed form, as well as to avoid infinite tangential stresses at the interface of the layers near the model end. The obtained results of calculations coincide qualitatively and quantitatively with the results of the experiment.
\end{abstract}

\section{Statement of problem}

In most experiments, the adhesive strength of the compounds is estimated using the simplest criterion - the average breaking stress $\tau_{\text {mid }}$. This value is calculated as the ratio of the breaking load $P$ to the bonding area $A, \tau_{\text {mid }}=P / A$. This method of evaluation involves a uniform stress distribution over the bonding area. However, as shown by the results of measuring the average adhesion strength, this value is a strong function of the geometric parameters of the tested models. This is a consequence of the fact that the stresses are distributed unevenly over the bonding area. In particular, in most experiments on the measurement of stresses in adhesion models, an edge effect was identified, i.e. a stress concentration similar to that observed at the tops of a crack.

The results of a detailed measurement of the stresses on the contact surface of a glass rod and an adhesive by polarization-optical method are given in [1]. The general view of the experimental model is presented below, in Figure 1. This sample, represented by three bonded rods, is loaded with a compressive force along the axis of the central rod. For the calculation we will apply the model with the use of a contact layer.

\footnotetext{
* Corresponding author: science@nikitatsybin.ru
} 


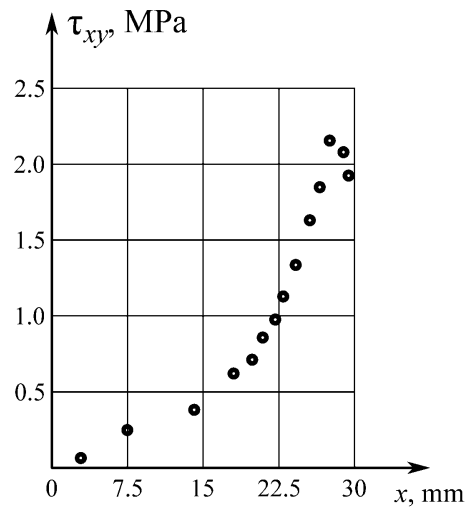

Fig. 1. Experimental model
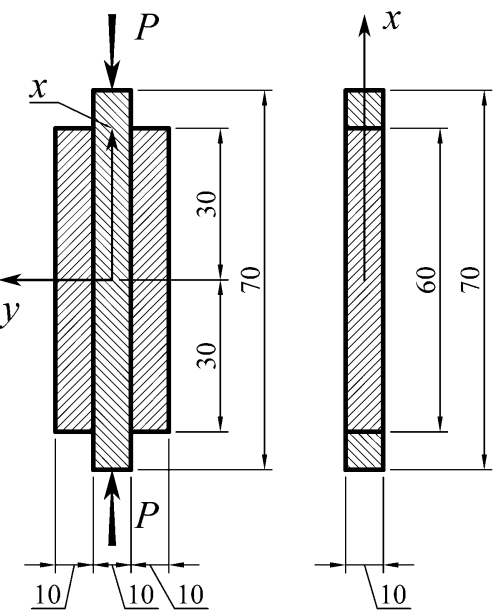

\section{Analytical solution of the problem}

In paper $[2,3]$ the resolving system of equations for a multilayer rod is presented with the use of the contact layer model. The general view of the model is shown in Figure 2.

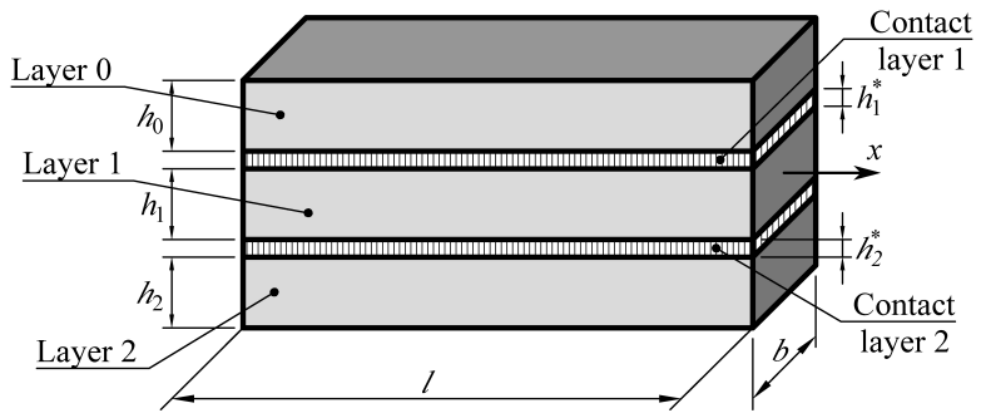

Fig. 2. Three-layer rod model

To obtain the resolving equations of a multilayer rod with contact layers, we need:

Navier's differential equations of equilibrium

$$
\frac{\partial \sigma_{x}}{\partial x}+\frac{\partial \tau_{x y}}{\partial y}=0 ; \frac{\partial \tau_{y x}}{\partial x}+\frac{\partial \sigma_{y}}{\partial y}=0 .
$$

Cauchy strain-displacement relations

Hooke's law

$$
\varepsilon_{x}=\frac{\partial u}{\partial x} ; \varepsilon_{y}=\frac{\partial v}{\partial y} \gamma_{x y}=\frac{\partial u}{\partial y}+\frac{\partial v}{\partial x} .
$$

$$
\varepsilon_{x}=\left(\sigma_{x}-\mu \sigma_{y}\right) / E ; \varepsilon_{y, k}=\left(\sigma_{y}-\mu \sigma_{x}\right) / E ; \gamma_{x y}=\tau_{x y} / G
$$

For layers we will use Euler-Bernoulli hypothesis: plane sections remain plane and normal to the deflected neutral axis. As result from equations (2) it is obtained

$$
\gamma_{x y, k}=0 \rightarrow u_{k}=u_{0, k}-y_{k} \frac{\partial v_{k}}{\partial x} \rightarrow \varepsilon_{x, k}=\varepsilon_{0 x, k}-y_{k} \frac{\partial^{2} v_{k}}{\partial x^{2}} .
$$


It is also assumed that the stress $\sigma_{y}$ in Hooke's law can be neglected.

$$
\sigma_{x, k}=E_{k}\left(\varepsilon_{0 x, k}-y_{k} \frac{\partial^{2} w_{k}}{\partial x^{2}}\right) \text {. }
$$

We replace the stresses $\sigma_{x}$ with statically equivalent internal forces by formulae

$$
N_{k}=\int_{-h_{k} / 2}^{h_{k} / 2} \sigma_{x, k} d y_{k} ; M_{k}=\int_{-h_{k} / 2}^{h_{k} / 2} \sigma_{x, k} y_{k} d y_{k} ; \sigma_{x, k}=E_{k}\left(\frac{N_{x, k}}{B_{k}}+y_{k} \frac{M_{k}}{D_{k}}\right) \text {. }
$$

To obtain the resolving equations for the layers, it is required to substitute the expression (6) in (1) and integrate, taking into account the boundary conditions.

$$
\tau_{x y, k}\left(-h_{k} / 2\right)=P_{x, k}^{t} ; \tau_{x y, k}\left(h_{k} / 2\right)=P_{x, b}^{t} ; \sigma_{y, k}\left(-h_{k} / 2\right)=P_{y, k}^{t} ; \sigma_{y, k}\left(h_{k} / 2\right)=P_{y, k}^{b} .
$$

Let us write the system of resolving equations [2] for the layers $0,1,2$

$$
\frac{d^{2} N_{k}}{d x^{2}}=\frac{d P_{x, k}^{t}}{d x}-\frac{d P_{x, k}^{b}}{d x} ;-D_{k} \frac{d^{4} v_{k}}{d x^{4}}+\frac{h_{k}}{2}\left(\frac{d P_{x, k}^{t}}{d x}+\frac{d P_{x, k}^{b}}{d x}\right)=P_{y, k}^{t}-P_{y, k}^{b}, k=0,1,2 .
$$

The values $P_{x, k}^{t}, P_{x, k}^{b}, P_{y, k}^{t}, P_{y, k}^{b}$ in (8) represent the shear and normal forces applied to the top and bottom faces of the layers $0,1,2$. In general case, these values represent the external loads applied to the model and the stresses arising in the contact layer. The values $B_{k}$ and $D_{k}$ are the integral stiffness of the layers for tension and bending $B_{k}=E_{k} b h_{k} ; D_{k}=E_{k} b h_{k}^{3} / 12$.

Consider the contact layer. Because the rods are not connected, in Hooke's law for an orthotropic body Young's modulus $E_{x, k}^{*}$ and Poisson ratio $\mu_{k}^{*}$ must be set equal to zero. As a result, the stresses $\sigma_{x, k}^{*}$ are zero. From the first equation (1) it follows that $\tau_{x y, k}^{*}$ is independent of the variable $y_{k}^{*}$. Here and further, all values related to the contact layer will be marked with a symbol *. From (3) we obtained $\sigma_{y, k}^{*}=E_{y, k}^{*} \partial v_{k}^{*} / \partial y_{k}^{*}$. We substitute this expression into the second equation (1)

$$
\frac{\partial^{2} v_{k}^{*}}{\partial\left(y_{k}^{*}\right)^{2}}=-\frac{1}{E_{y, k}^{*}} \frac{d \tau_{x y, k}^{*}}{d x} .
$$

Let us integrate (9) with the boundary conditions $v_{k}^{*}\left(-h_{k}^{*} / 2\right)=v_{t, k}^{*} ; v_{k}^{*}\left(h_{k}^{*} / 2\right)=v_{b, k}^{*}$.

$$
v_{k}^{*}=-\frac{1}{2 E_{y, k}^{*}}\left[\left(y_{k}^{*}\right)^{2}+\frac{\left(h_{k}^{*}\right)^{2}}{4}\right] \frac{d \tau_{x y, k}^{*}}{d x}-\frac{y_{k}^{*}}{h_{k}^{*}}\left(v_{t, k}^{*}-v_{b, k}^{*}\right)+\frac{1}{2}\left(v_{t, k}^{*}+v_{b, k}^{*}\right) .
$$

We substitute the expression (10) in the first equation (1). By integrating twice with respect to $y_{k}^{*}$ with allowance for the boundary conditions $u_{k}^{*}\left(-h_{k}^{*} / 2\right)=u_{t, k}^{*} ; u_{k}^{*}\left(h_{k}^{*} / 2\right)=u_{b, k}^{*}$

$$
\begin{aligned}
u_{k}^{*}=\frac{y_{k}^{*}}{6 E_{z, k}^{*}}\left[\left(y_{k}^{*}\right)^{2}\right. & \left.-\frac{\left.\left(h_{k}^{*}\right)^{2}\right]}{4}\right] \frac{d^{2} \tau_{x y, k}^{*}}{d x^{2}}+\frac{4\left(y_{k}^{*}\right)^{2}-\left(h_{k}^{*}\right)^{2}}{8 h_{k}^{*}}\left(\frac{d v_{t, k}^{*}}{d x}-\frac{d v_{b, k}^{*}}{d x}\right)- \\
& -\frac{y_{k}^{*}\left(u_{t, k}^{*}-u_{b, k}^{*}\right)}{h_{k}^{*}}+\frac{1\left(u_{t, k}^{*}+u_{b, k}^{*}\right)}{2} .
\end{aligned}
$$

The system of two differential equations with respect to the stresses in the contact layers can be obtained by substituting the expressions (10) and (11) in equations (3). 


$$
\frac{\tau_{k}^{*}}{G_{k}^{*}}=\frac{\left(h_{k}^{*}\right)^{2}}{12 E_{k}^{*}} \frac{d^{2} \tau_{k}^{*}}{d x^{2}}+\frac{1}{2} \frac{d\left(v_{t, k}^{*}+v_{b, k}^{*}\right)}{d x}-\frac{u_{t, k}^{*}-u_{b, k}^{*}}{h_{k}^{*}} ; \sigma_{k}^{*}=-y_{k}^{*} \frac{d \tau_{k}^{*}}{d x}-\frac{E_{k}^{*}\left(v_{t, k}^{*}-v_{b, k}^{*}\right)}{h_{k}^{*}}, k=1,2 .
$$

In these equations $E_{y, k}^{*}=E_{k}^{*}, G_{x y, k}^{*}=G_{k}^{*}=E_{k}^{*} / 2$. Values $v_{t, k}^{*}, v_{b, k}^{*}, u_{t, k}^{*}, u_{b, k}^{*}$ represent the displacements of the top and bottom external layers and are determined from expressions

$$
u_{t, k}^{*}=u_{0, k-1}-\frac{h_{k-1}}{2} \frac{d v_{k-1}}{d x} ; u_{b, k}^{*}=u_{0, k}+\frac{h_{k}}{2} \frac{d v_{k}}{d x} ; v_{t, k}^{*}=v_{k-1} ; v_{b, k}^{*}=v_{k}, k=1,2
$$

Let us consider the values more detailed $P_{x, k}^{t}, P_{x, k}^{b}, P_{y, k}^{t}, P_{y, k}^{b}$. In our case, in accordance with Figure 1, 2 we obtain

$$
\left.\begin{array}{l}
P_{x, 0}^{t}=P_{y, 0}^{t}=0 ; P_{x, 0}^{b}=\tau_{1}^{*} ; P_{y, 0}^{b}=\sigma_{1}^{*}\left(-h_{1}^{*} / 2\right) ; P_{x, 1}^{t}=-\tau_{1}^{*} ; P_{y, 1}^{t}=\sigma_{1}^{*}\left(h_{1}^{*} / 2\right) ; \\
P_{x, 1}^{b}=\tau_{2}^{*} ; P_{y, 1}^{b}=\sigma_{2}^{*}\left(-h_{2}^{*} / 2\right) ; P_{x, 2}^{t}=-\tau_{2}^{*} ; P_{y, 2}^{t}=\sigma_{2}^{*}\left(h_{2}^{*} / 2\right) ; P_{x, 2}^{b}=P_{y, 2}^{b}=0 .
\end{array}\right\}
$$

The following relations can be obtained from the symmetry condition of the problem with respect to the axis $x$

$$
\left.\begin{array}{c}
N_{2}=N_{0} ; N_{1}=P-2 N_{0} ; \tau_{2}^{*}=-\tau_{1}^{*} ; v_{1}=0 ; \\
v_{2}=-v_{0} ; u_{2}=u_{0} ; h_{2}=h_{0} ; B_{2}=B_{0}=E_{0} b h_{0} ; D_{2}=D_{0}=\frac{E_{0} b h_{0}^{3}}{12}
\end{array}\right\}
$$

System of equations (8) and (12), using expressions (13), (14), (15) can be reduced to one differential equation with respect to the longitudinal force $N_{0}$

$$
\frac{d^{8} N_{0}}{d x^{8}}-\omega \frac{d^{6} N_{0}}{d x^{6}}+\omega \lambda \chi \frac{d^{4} N_{0}}{d x^{4}}-\omega \lambda \frac{d^{2} N_{0}}{d x^{2}}+\omega \lambda \eta \xi N_{0}=\omega \lambda \xi P,
$$

in which

$$
\omega=\frac{24}{\left(h^{*}\right)^{2}} ; \lambda=\frac{2 G^{*}}{h^{*} D_{0}} ; \chi=\frac{h_{0}^{2}}{6}+\frac{h_{0} h^{*}}{4}+\frac{\left(h^{*}\right)^{2}}{6}+\frac{D_{0}}{B_{1}} ; \eta=2+\frac{B_{1}}{B_{0}} ; \xi=\frac{G^{*}}{B_{1} h^{*}} .
$$

The moments and transverse forces $M_{0}, Q_{0}$, acting in layer 0 , as well as tangential stresses $\tau_{1}^{*}$ in the contact layer, can also be expressed through a longitudinal force $N_{0}$

$$
\left.\begin{array}{c}
\tau_{1}^{*}=-\frac{d N_{0}}{d x} ; M_{0}=\frac{4}{\left(h_{0}+h^{*}\right) \lambda}\left(-\frac{1}{\omega} \frac{d^{4} N_{0}}{d x^{4}}+\frac{d^{2} N_{0}}{d x^{2}}-\eta \xi N_{0}+\xi P\right) ; \\
Q_{0}=\frac{4}{\left(h_{0}+h^{*}\right) \lambda}\left(-\frac{1}{\omega} \frac{d^{5} N_{0}}{d x^{5}}+\frac{d^{3} N_{0}}{d x^{3}}-\eta \xi \frac{d N_{0}}{d x}\right)+\frac{h_{0}}{2} \frac{d N_{0}}{d x} .
\end{array}\right\}
$$

As a result of integrating equation (16), we obtain eight unknown constants. Since the problem is symmetric with respect to the axis $y$, there are only four unknowns. Four boundary conditions are required to determine them.

It is also necessary to inform that in this case we have included the adhesive layers in the contact layer. This is acceptable [10], since the adhesive layers are thin enough, and their rigidity is negligible compared to the rod stiffness. 


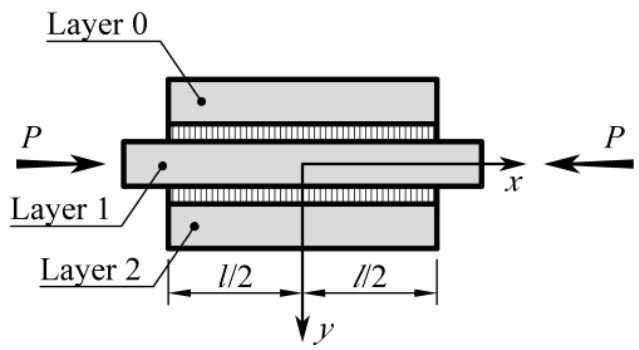

Fig. 3. To the statement of boundary conditions

The boundary conditions for the solution of this problem (Figure 3), taking into account the symmetry with respect to the axis $y$, are written below

$$
N_{0}(l / 2)=M_{0}(l / 2)=0 ; Q_{0}(l / 2)=\tau_{1}^{*}(l / 2)=0 .
$$

According to the symmetry, the solution of equation (16) takes the form

$$
N_{0}=\frac{P}{\eta}+\sum_{m=1}^{4} C_{m} \cosh \left(\sqrt{s_{m}} x\right)
$$

in which the values $s_{m}$ are the roots of the characteristic equation

$$
s_{m}^{4}-\omega s_{m}^{3}+\omega \lambda \chi s_{m}^{2}-\omega \lambda s_{m}+\omega \lambda \eta \xi=0 .
$$

Further, we will use the following mechanical and physical-geometric parameters in calculations

$$
\begin{aligned}
& h_{0}=h_{1}=h_{2}=b=10 \mathrm{~mm} ; h_{1}^{*}=h_{2}^{*}=2 \mathrm{~mm} ; l=60 \mathrm{~mm} ; \\
& G^{*}=15000 \mathrm{MPa} ; E_{0}=E_{1}=70000 \mathrm{MPa} ; P=0.6 \mathrm{kN} .
\end{aligned}
$$

\section{Modelling the problem using FEM without a contact layer. Singularity of tangential stresses}

In confirmation of the fact that the solution of this problem in the case of ideal contact of the layers leads to the appearance of the stress singularity, a series of calculations is performed in the software package Lira-Soft. In modelling (due to symmetry) to accelerate the calculation, a quarter of the sample is considered. The problem is solved in a linear formulation. To obtain more adequate results, the mesh is thickened at the angular point of the model. Below is a general view of the model (Figure 4).

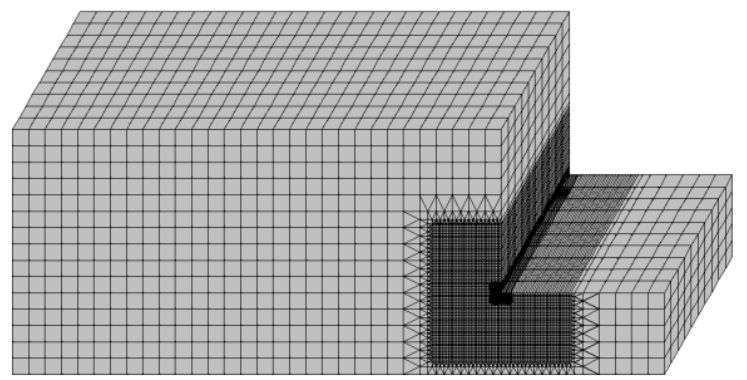

Fig. 4. General view of FEM model

The results of the calculations showed that in the ideal contact of the layers, in spite of the boundary conditions of the problem, stress concentration zones arise at the corner points on load-free surfaces. Isolines of tangential stresses at the corner point of the model are presented in Figure 5. 


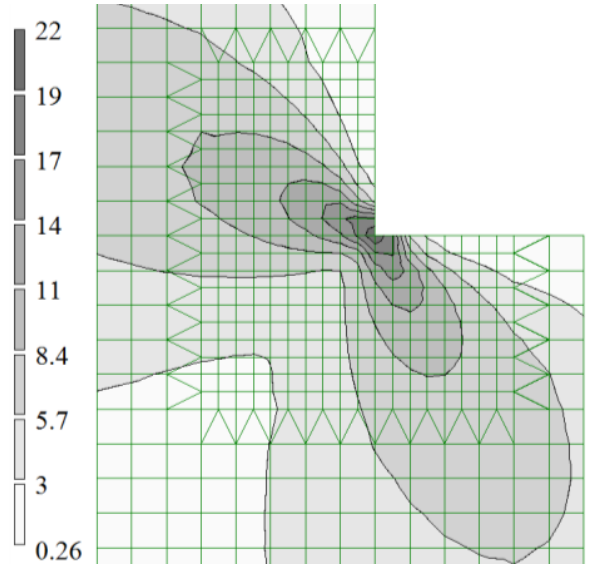

Fig. 5. Isolines of tangential stresses at the corner point of the model

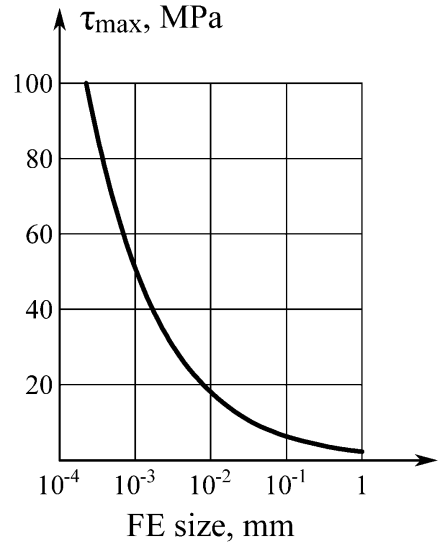

Fig. 6. Dependence of the max value of the tangential stresses on the size of the FE mesh

It was found that the value of the maximum tangential stresses increases with decreasing size of the finite element mesh of the model. The results of the calculation of the maximum tangential stresses at the end of the model at the interface of the rods are shown in Figure 6. We remind that in reality at this point the tangential stresses are zero.

As we can see, as the mesh decreases and the FEM calculation approaches the analytical solution, the maximum stress value increases and tends to singularity. Thus, the correct choice of the model splitting grid in the calculation of adhesion compounds using FEM is one of the key tasks. It is worth noting that the solution of this problem in a physically nonlinear formulation will avoid infinite stresses, but the calculation speed will be significantly reduced.

\section{Comparison of the results of analytical calculation, FEM calculation with the contact layer and the experiment}

The results of FEM calculation (obtained in the previous section) showed that under the conditions of ideal contact of rods the stress singularity effect is manifested. This fact does not allow a qualitative comparison of the FEM model with the analytical calculation and experimental results. Therefore, first, we will perform a numerical calculation of the model from the previous section by adding a contact layer. The FEM model of a three-layer rod with a contact layer is given below on Figure 7. In Figure 7, the contact layer is represented by darkened elements. As before, the mesh point is thickened at the corner point.

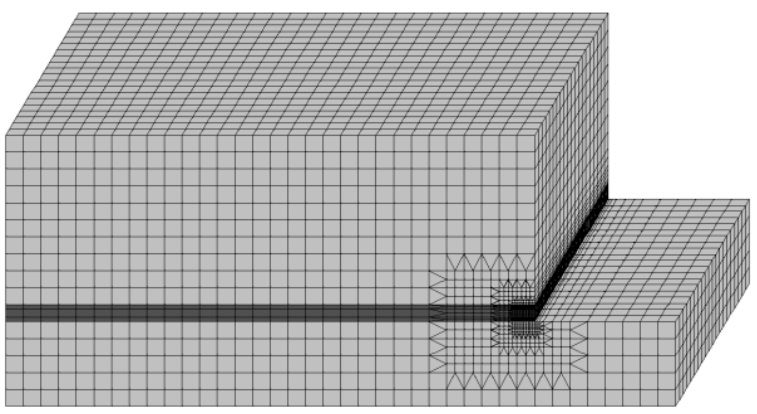

Fig. 7. General view of FEM model with contact layer 
After adding a contact layer to the finite element model, the influence of the finite element mesh size (Figure 9) on the stress-strain state of the model was analysed.

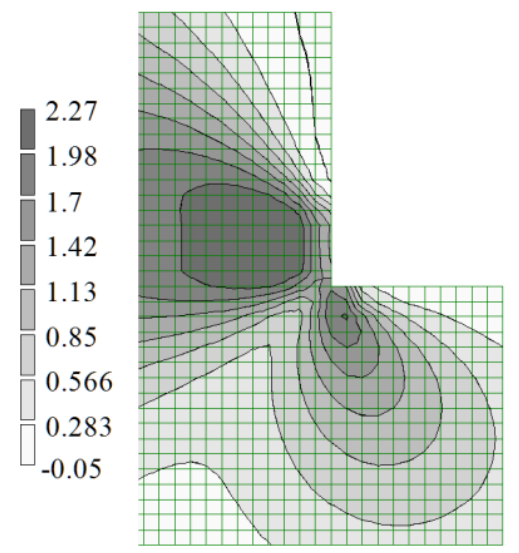

Fig. 8. Isolines of tangential stresses at the corner point of the model with a contact layer

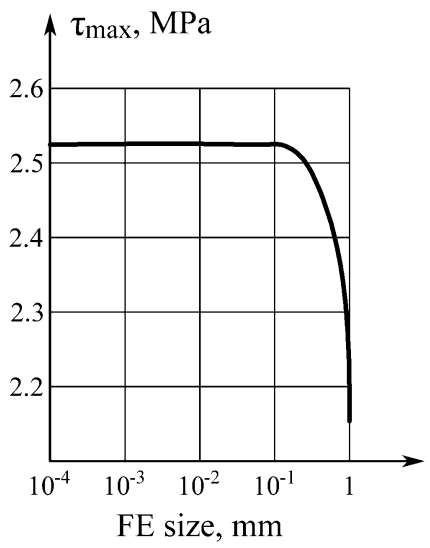

Fig. 9. Dependence of the max value of the tangential stresses in a contact layer model on the size of the FE grid

As we can see, the use of the contact layer model for describing the adhesion interaction between the layers in FEM calculation allows to avoid infinite tangential stresses (Figure 8) at the corner point. The comparison of the results of analytical and numerical calculations with the experimental results is shown in Figures 10 and 11. Numerical calculation was made on the mesh size of $0.2 \times 0.2 \mathrm{~mm}$.

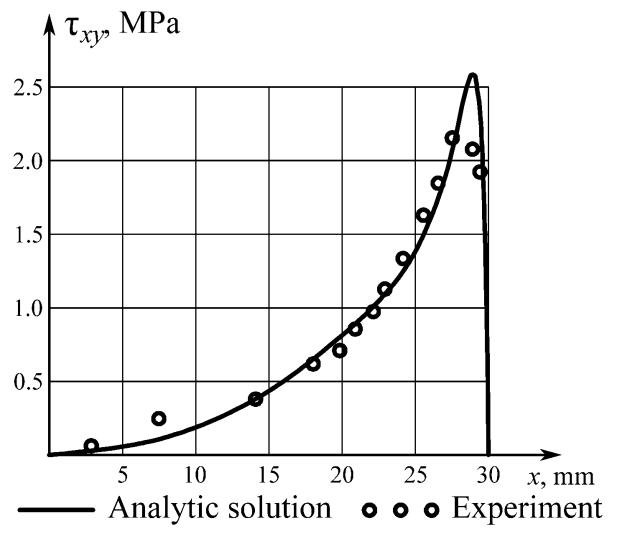

Fig. 10. Comparison of analytical calculation results with experimental data

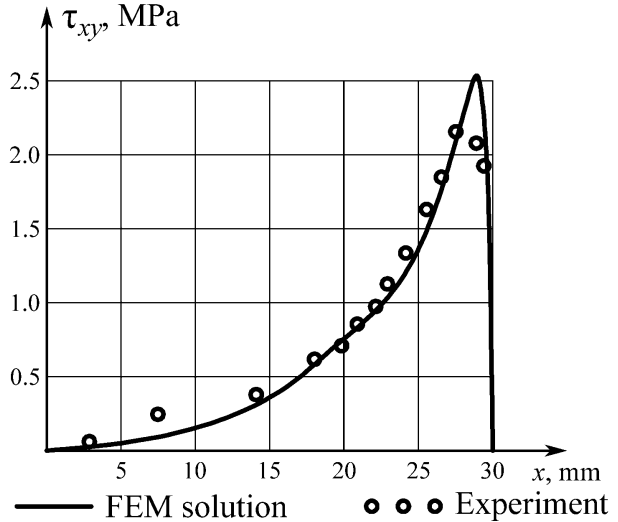

Fig. 11. Comparison of numerical calculation results with experimental data

As we can see, the results of the calculation are in qualitative agreement with the experimental data. It is not possible to provide the results of numerical and analytical calculations on one graph, as the curves almost completely coincide.

\section{Conclusion}

The paper compares the results of an analytical and numerical calculation of a three-layer rod under central compression with experimental results. It is demonstrated that the use of the contact layer model in analytical and numerical calculations allows analysis of the essentially inhomogeneous stress-strain state of multilayer rods. The obtained results are 
consistent with the experimental data. In addition, it should be noted that the proposed model of adhesion interaction avoids the singularity of stresses at the corner points, and also allows satisfying all boundary conditions. The results of the analysis of numerical calculation of the model using FEM allow to determine the size of the finite element grid, in order to obtain adequate results in other problems.

This work was financially supported by the Ministry of Russian Education (state task \#7.1524.2017/6.7)

\section{References}

1. A.V. Turazyan, A.L. Rabinovich, Reports of the Academy of Sciences of the USSR, 191(6), 1305 (1970)

2. V.I. Andreev, R.A. Turusov, N. Yu. Tsybin, MATEC Web of Conferences, 117 (2017)

3. V.I. Andreev, R.A. Turusov, N. Yu. Tsybin, Procedia Engineering, 153, 59 (2016)

4. N.Yu. Tsybin, R.A. Turusov, V.I. Andreev, Procedia Engineering, 153, 51 (2016)

5. V.I. Andreev, R.A. Turusov, N.Yu. Tsybin, Advances in Engineering Research, Atlantis Press, 39, 1703 (2015)

6. A.M. Kuperman, R.A. Turusov, A.J. Gorenberg, V.I. Solodilov, R.A. Korokhin, Y.A. Gorbatkina, V.G. Ivanova-Mumzhieva, O.A. Zhuravleva, A.V. Baikov, Mechanics of Composite Materials, 50(6),705 (2015)

7. R.A. Turusov, A.M. Kuperman, Polymer Science. Series D, 7(1), 1 (2014)

8. R.A. Turusov, K.T. Vuba, A.S. Freidin, Proceedings of TSNIISK n.a. V. A. Koucherenko, 24, 86 (1972)

9. A.S. Freidin, R.A. Turusov, Properties and calculation of adhesive joints. (Publ. House Chemistry, Moscow, 1990)

10. R.A. Turusov, Adhesive mechanics. (Publ. House of MGSU, Moscow, 2016)

11. T. Hayashi, Photoelastsche Untersuchungen der Spannungs-Verteilung in der durch Fasernverstarkten Platte. Nonhomogenity and Plasticity. (Publ. House Hill, New York, 1959)

12. V.I. Andreev, R.A.Turusov, N.Yu. Tsybin, IOP Conf. Ser.: Mater. Sci. and Eng., 365, 042049 (2018) 